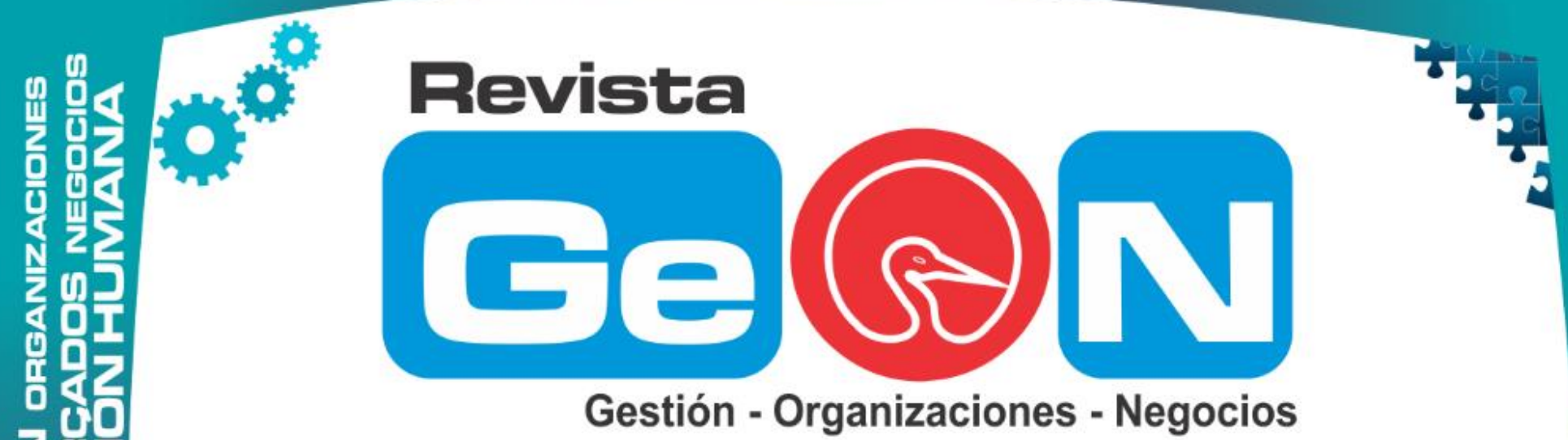

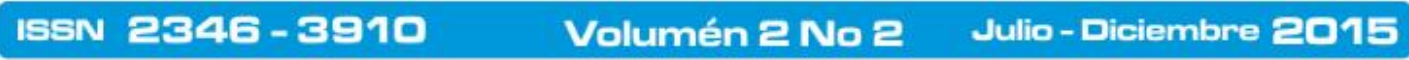

Revista Electrónica de la Facultad de Ciencias Económicas de la Universidad de los Llanos

1. 3 II

440

VILLAVICENCIO - COLOMBIA

$>2$

64

2

2 平品

70

ব)

展

o

ii III

0 U

ใด

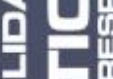

居的

임

Iiin in

照

只

造

iil 2

if in

4 吕

2

400

付 iI
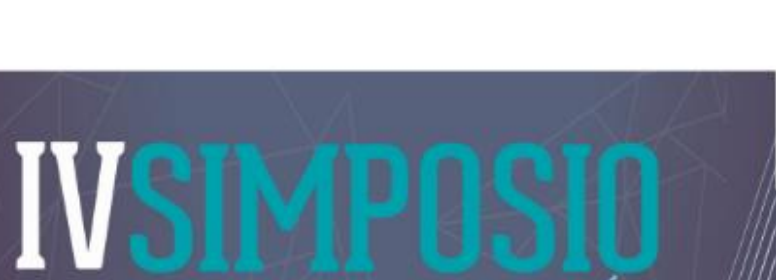

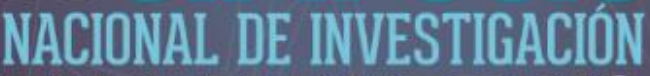

EN MARKETING
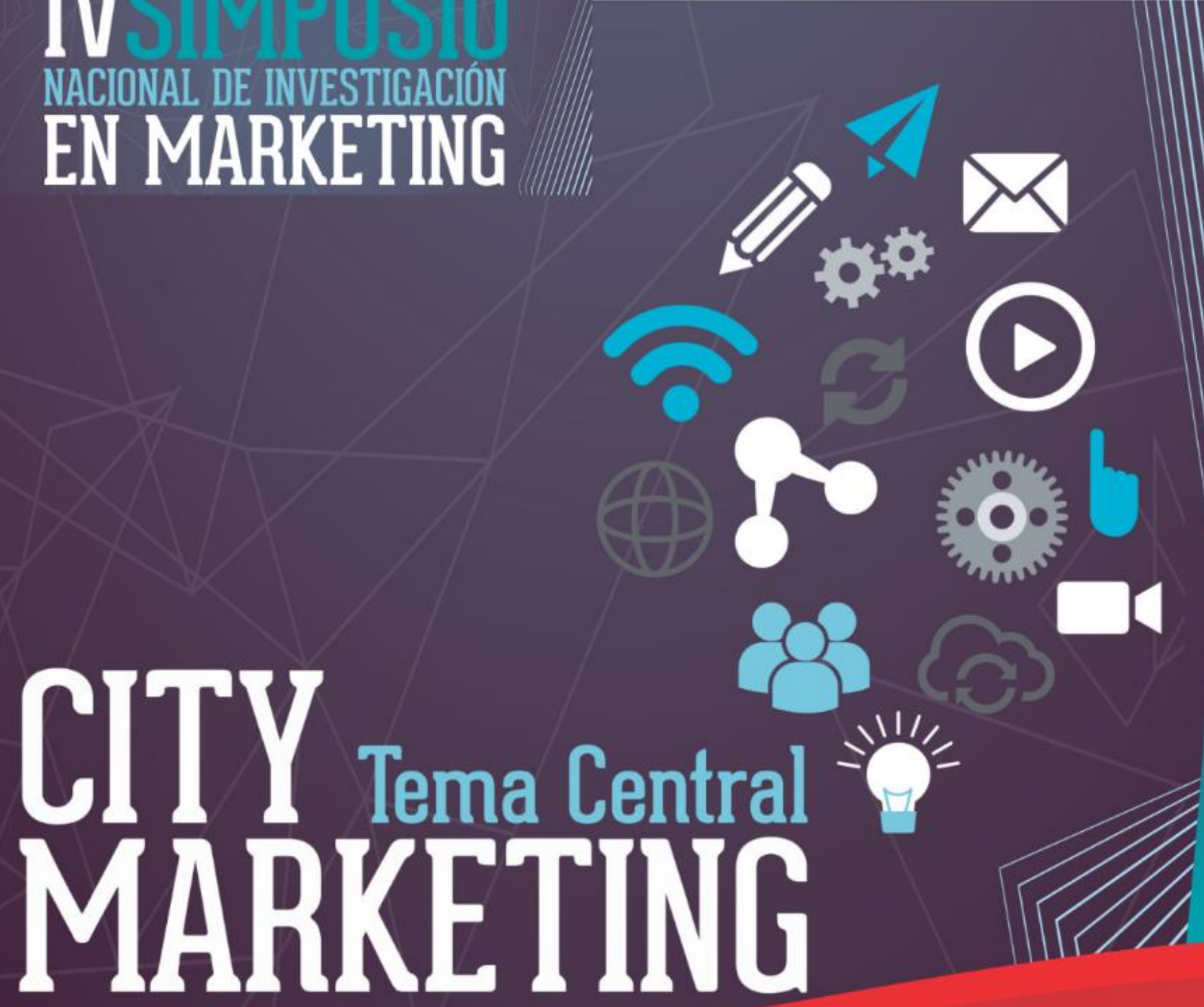

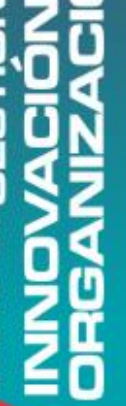
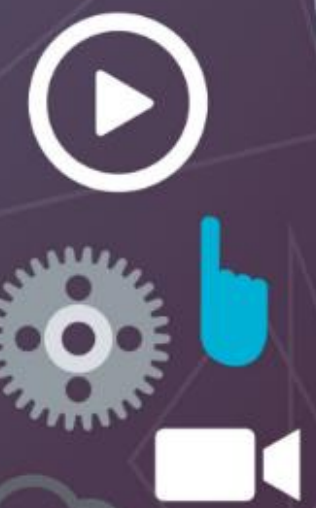


\section{Marca Joropo - Erika Baquero - Ángela Tovar - Guillermo Quiñonez - Jennifer Vega}

Erika Ximena Baquero Urrego

Estudiante de Negocios Internacionales. Universidad Santo Tomás

erikabaquero@usantotomas.edu.co

Ángela Patricia Tovar Macías

Estudiante de Negocios Internacionales.

Universidad Santo Tomás

angela.tovar@usantotomas.edu.co

Guillermo Alejandro Quiñónez

Magister en Dirección y administración de empresas

guillermoquinonez@usantotomas.edu.co

Jennifer Vega Barbosa

Magister en Publicidad

jennifervega@usantotomas.edu.co

\section{Resumen}

El presente artículo plantea algunos avances sobe la investigación, por medio del cual se evalúa la posibilidad de crear una marca que presente al folclor llanero en el exterior. El objetivo general de la investigación consistió en identificar los atributos y los factores del folclor llanero que se deben potencializar, para que en el futuro sirva de base para su internacionalización por medio de una marca. La metodología utilizada tuvo un enfoque mixto, con el fin de obtener un panorama más amplio y profundo.

La investigación "Marca Joropo" estuvo sujeto a teorías sobre la internacionalización la marca y el folclor llanero. En cuanto a la internacionalización se decidió implementar la teoría ecléctica, así como el modelo UppSala, ya que se pretende aplicar la teoría de Born Global a la marca deseada. Por otro lado, el concepto de marca más idóneo, es el que plantea Kevin Roberts en su libro Lovemarks (2005), en lo relacionado con el folclor llanero se trabajó con el libro del Folclor llanero, de Miguel Ángel Martin (2000).

\section{Palabras clave}

Joropo; Folclor Llanero; Marca; internacionalización.

\begin{abstract}
This paper presents some progress on research, whereby the possibility of creating a brand that represents the Ranger folklore abroad is evaluated. The overall objective of the research was to identify attributes and factors that must Ranger potentiate folklore, so that in the future as a basis for internationalization through a brand. The methodology was a mixed approach, in order to get a broader and deeper picture.
\end{abstract}

The research "Brand Joropo" was subject to theories of internationalization brand and burrowing folklore. As for internationalization it was decided to implement the eclectic theory and the Uppsala model, as it is intended to apply the theory of Born Global to the desired mark. On the other hand, the concept most suitable brand, is posed by Kevin Roberts in his book Lovemarks (2005), as it relates to burrowing folklore worked with Ranger's book Folklore, Miguel Angel Martin (2000)

\section{Keywords}

Joropo; plains folklore; brand; internationalization.

\section{Introducción}

Al observar el atractivo que tienen los llanos orientes y su potencial para atraer turistas y simpatizantes, no solo nacionales sino de nivel 
internacional, se ve la necesidad de estudiar más a fondo las ventajas que tiene la región con la riqueza cultural y las oportunidades que podrían presentarse si se explota esta ventaja. Teniendo en cuenta que hay gran diversidad en cuanto al folclor llanero y con ello grandes representantes que dan muestra del potencial que se tiene, florece la pregunta de investigación la se cuestiona cuáles son los factores que se deben potencializar del folclor llanero para lograr su internacionalización por medio de una marca.

Para la buena realización de la investigación y apoyar el estudio realizado, se fundamenta mediante la elaboración de un marco teórico, el cual nos permite delimitar el problema, mediante la documentación de las bases teorías propuestas para la internacionalización, la marca y el folclor llanero.

En cuanto a la internacionalización se decide implementar la teoría ecléctica de Dunning en1980, citado por (Marroquin \& Castroman) en cuanto a las ventajas propias que tiene el producto a investigar (folclor llanero), así como el modelo UppSala (Johanson \& Vahlne, 1977) citado por (Brenes \& Darner, 2008), ya que se visualiza aplicar la teoría de Born Global a la marca deseada, porque se enfocó que el resultado de la "Marca Joropo" inicie en un mercado internacional.

Para la marca, se determina que la definición más pertinente, es el enfoque que plantea Roberts (2005), en su libro Lovemarks, ya que habla de la capacidad que tiene un producto de diferenciarse de otro, y cuya solución no solo se basa en la creación de un producto, sino en experiencias capaces de crear vínculos emocionales y duraderos con sus consumidores.

Para el folclor llanero se decide tomar como base de investigación al libro "Del folclor llanero" de Miguel Ángel Martín (2000) ya que encierra todo lo que comprende el folclor de los llanos. Así mismo, se cita al historiador y folclorista Jairo Ruiz Churrión (2013) por su interpretación acerca del folclor llanero.
Con base en el interrogante, se realiza una investigación minuciosa, con la que se cumple el objetivo general el cual busca identificar los atributos que tiene el folclor llanero para lograr su internacionalización, por medio de una marca. Así como los objetivos generales, los cuales se resumen en conocer e identificar las fortalezas y debilidades del folclor llanero, y así mismo determinar los aspectos necesarios que debe tener la marca para que se adapte al mercado global, partiendo desde la hipótesis de que el folclor llanero tiene las cualidades necesarias para la creación de una marca con potencial de internalización, si se le da el enfoque adecuado.

\section{Desarrollo}

Esta investigación tiene un enfoque mixto, ya que se recolectaron y analizaron datos de manera cuantitativa y cualitativa con el fin de obtener un panorama más amplio y profundo del estudio de la investigación.

En el enfoque cuantitativo, el tipo de investigación es la No Experimental, Transversal y Exploratoria, ya que es un estudio que no se ha investigado, o si se ha realizado es muy limitado.

Para dar cumplimiento con el objetivo planteado, la investigación cuantitativa se realizó a través de la aplicación de 43 encuestas propias, de las cuales 23 fueron dirigidas a extranjeros y 20 a personas que conocen el folclor llanero.

Hay que aclarar que la aplicación de estos instrumentos se hizo como prueba piloto, ya que esta investigación se ampliará, por medio del semillero de investigación Interbranding, el cual hace parte de la facultad de Negocios Internacionales.

En el enfoque cualitativo, el tipo de investigación es la Etnográfica, ya que es una investigación cultural. Esta se realizó a través de la aplicación de 5 entrevistas a diferentes folcloristas de renombre que son promotores $y$ exponentes de la tradición llanera y buscan a la vez su desarrollo y crecimiento. 
Para cumplir con el objetivo de la investigación se aplicaron los instrumentos mencionados anteriormente los cuales muestran lo siguiente. En el enfoque cuantitativo, las encuestas que iban dirigidas a las personas conocedoras del joropo, arrojaron como resultado que el $70 \%$ de los encuestados están totalmente de acuerdo en que el joropo tiene los atributos necesarios para ser internacionalizado.

Para identificar los principales componentes de una muestra folclórica, se les preguntó cuáles factores consideran importantes en una representación folclórica, lo que dio como resultado que el baile es el factor más primordial ya que el $80 \%$ de los encuestados lo incluyeron en la lista, así mismo, la música está en la lista con un $75 \%$, seguido por la gastronomía, los trajes típicos y los cantos con el 60,55 y 40 por ciento respectivamente.

Uno de los puntos más importantes, es la perspectiva que tienen las personas a cerca de lo que significa la palabra joropo y que factores del folclor llanero la encierran. En este sentido, el $50 \%$ de los encuestados coincidieron en que el baile es el factor más importante para identificar el joropo, seguido del canto, los instrumentos $\mathrm{y}$ el trabajo de llano seleccionado por 5 de las personas encuestadas cada uno.

En cuanto a los factores del joropo que deben mostrarse en el exterior, los factores más relevantes arrojados por las encuesta son que 1 de cada 2 encuestados afirmó que es el baile es el principal, y 2 de cada 5 personas menciono el canto como factor clave para mostrar en el exterior.

Algo relevante, es que los conocedores prefieren el joropo de espectáculo por encima del joropo tradicional con un $65 \%$ sobre un $35 \%$ del total de encuestados.

A lo referente a la creación de la Marca Joropo, el 50\% está de acuerdo, y el $40 \%$ de los encuestados están totalmente de acuerdo en que es una buena estrategia para la internacionalización del folclor llanero.
Y finalmente, 20 de 20 encuestados consideran que el joropo es una herramienta para atraer turistas extranjeros a la región. Por otro lado, el resultado de la encuesta dirigida a los extranjeros fue el siguiente. Los países desde donde respondieron la encuesta fueron, Estados Unidos, Canadá, México, Panamá, Venezuela, Chile, Argentina y España.

Al afirmar que las muestras folclóricas son apreciadas en esos países, 2 de los 23 encuestados estuvieron en desacuerdo, el 34,8\% son imparciales, y el $26,1 \%$ están de acuerdo y totalmente de acuerdo.

El 47,8\% de los encuestados son imparciales en cuanto a la preferencia entre las representaciones. Además, 8 de los encuestados afirma que están de acuerdo y completamente de acuerdo con que prefieren las representaciones culturales tradicionales sobre las actuales.

En cuanto a los factores importantes en una muestra folclórica, la música encabeza la lista ya que un $91,3 \%$ de los extranjeros, la incluyeron en la lista, así mismo, la gastronomía, el baile y los trajes típicos fueron resaltados con un $62,5 \%, 56,5 \%$ y $52,2 \%$ respectivamente.

Así mismo, los factores menos relevantes de una muestra folclórica para los extranjeros son la tradición oral y escrita, así como los mitos y leyendas de una cultura.

En cuanto al joropo, 17 de los encuestados, que representan el 73,9\% de los encuestados conocen de qué se trata. A este porcentaje, se le pregunto qué factores de la cultura llanera encierra la palabra joropo, de los cuales el canto, el baile y los instrumentos fueron los más representativos.

Se observa que 10 de los extranjeros que conocen el joropo, prefieren el joropo tradicional sobre el joropo de espectáculo. Y finalmente, el 95,7\% de los encuestados, afirmaron que están interesados en conocer más acerca del joropo.

En el enfoque cualitativo se seleccionó no aleatoriamente, en la ciudad de Villavicencio, a 
un grupo de folcloristas y promotores del folclor llanero. Se realizaron entrevistas semiestructuradas y se seleccionó la muestra teniendo en cuenta el grado de importancia, los conocimientos y experiencias vividas por parte de cada uno de los entrevistados.

De acuerdo con cada una de los relatos expuestos por los folcloristas se encontró lo siguiente. El $80 \%$ de los entrevistados manifiestan que las fortalezas del folclor llanero, son cada uno de los aspectos que lo componen, desde la música, baile, gastronomía, flora, fauna, literatura y su territorio; por otra parte el $20 \%$ sostuvo que las fortalezas se centran solo en el joropo tradicional: música, en cuanto a melodías, composiciones e instrumentos y el baile abarcando el traje y los movimientos.

De los cinco entrevistados, dos expresaron que una de las mayores limitaciones y debilidades que presenta el folclor llanero, es la falta de recursos económicos, y apoyo por parte de los entes gubernamentales y universidades, por otro lado dos exponentes más mencionaron que la falta de investigación y estudio del joropo y el folclor en general, son una de las falencias que se viven, así como la perdida de la tradición de la música, baile, costumbres y trajes.

De todos los aspectos que encierran la cultura llanera, el $100 \%$ de los folcloristas consideran que todos son importantes, pero si se habla de la construcción de una marca, los atributos que trasmiten la esencia y que generarían mayor impacto del folclor llanero a nivel internacional, son las expresiones artísticas tales como el canto, el baile y la composición.

Para identificar los principales factores que se deben potencializar del folclor llanero entre los exponentes y promotores de la cultura llanera el $80 \%$ afirmó que se debe estudiar e investigar cada uno de los aspectos de manera detallada, sin embargo, el $20 \%$ está de acuerdo con ese argumento, pero manifiesta que se debe trabajar más en los valores y en la formación de los artistas y buscar la unidad entre el gremio de folcloristas, para que el folclor llanero trascienda $\mathrm{y}$ se posicione a nivel nacional e internacional.

En las entrevistas los cinco folcloristas están interesados, en colaborar con la construcción de una marca que represente el Folclor llanero en el mundo.

\section{Conclusiones}

Con base en los resultados obtenidos, se considera que se comprueba la hipótesis propuesta.

De los resultados, se puede deducir que el folclor llanero, en especial el joropo, tiene los atributos necesarios para internacionalizarlo por medio de una marca comercial, ya que los folcloristas, por medio de sus experiencias han percibido como las raíces llaneras tienen la virtud de ser acogidas no solo en la región, sino de manera positiva han alcanzado escenarios nacionales e internacionales, dejando muestra de ello en cada una de sus presentaciones.

A partir de la percepción que tienen las personas de la región sobre el joropo, se puede analizar que este tiene las cualidades necesarias para la creación de la marca propuesta, ya que el $70 \%$ de los encuestados está totalmente de acuerdo y el $30 \%$ restante de acuerdo en que el folclor de los llanos orientales de Colombia se puede llevar de manera positiva al exterior.

Así mismo, al observar que solo el $52.2 \%$ de los extranjeros encuestados conocen la representación folclórica de los llanos se confirma lo dicho por Baquero (1990) cuando expone que el folclor llanero no cuenta con un reconocimiento ni con una conexión cultural profunda a nivel nacional y menos con el exterior. Pero a pesar del poco esfuerzo de difusión que ha hecho el gobierno regional, y los ejecutores del folclor para promoverlo a nivel internacional, el 73,9\% de los encuestados tienen conocimiento de lo que es el joropo, y empatía con las manifestaciones artísticas de la cultura llanera, y el hecho de que el $95,7 \%$ de los encuestados extranjeros afirmaran que están interesados en conocer más acerca del joropo, 
comprueba que tiene las cualidades necesarias para adaptarse y ser acogido en mercados externos.

Por otro lado, a partir de lo observado en la investigación, se considera que todas las representaciones culturales del folclor llanero son importantes y podrían llevarse al exterior, tal como se evidencia en los resultados de las encuestas aplicadas, en donde se resalta que tanto la música, el baile, la gastronomía, los trajes típicos, los cantos y demás factores son importantes en una muestra folclórica, lo que es congruente con lo afirmado por Jairo Ruiz Churrion (2013) cuando dijo que el folclor llanero se caracteriza por la esencia que trasmite el llanero a través del baile, canto, costumbres, gastronomía, flora, fauna, literatura y el territorio.

Uno de los principales hallazgos de esta investigación es la plena identificación de las fortalezas y las debilidades actuales del folclor llanero, que debido a la carencia de un estudio pertinente del tema y una investigación que documentara los atributos y falencias que presenta, no se cuenta la información necesaria para la creación de la marca planteada.

Por los resultados obtenidos de las entrevistas, se considera que las fortalezas del folclor de los llanos son uno de los puntos clave para su internacionalización, las cuales le dan valor a la muestra. Según los folcloristas que conocen a fondo la situación que vive la cultura llanera, con sus antecedentes y una evidente evolución, las fortalezas propias del folclor son todos los elementos que lo integran que parte desde el joropo tradicional con el baile, la música, la composición, los ritmos como el seis por derecho y el pajarillo, el canto y los instrumentos, en especial el arpa ya que hace reaccionar a la gente de manera increíble.

De igual manera se derivan las costumbres, los trajes típicos, la gastronomía, el trabajo de llano y la ganadería. No obstante dos de los entrevistados enfatizaron que las fortalezas del folclor se centran en el joropo de espectáculo sin dejar lo tradicional claramente, ya que por medio de esta mezcla, se ha logrado hacer varias giras en el exterior, generando gran impacto $\mathrm{y}$ acogida en el público.

Por otra parte uno de los folcloristas destaco que la principal fortaleza es la música llanera, porque es el elemento que genera mayor calidad y convoca a que muchos artistas se inclinen por este tipo de expresión.

En otro sentido, las entrevistas arrojaron ciertas falencias que presenta el folclor llanero, las cuales se considera que se deben tener en cuenta para que en futuras investigaciones $o$ esfuerzos que se realicen por parte del gobierno o las entidades culturales, se trabaje en su fortalecimiento, lo que permitirá darle una mayor proyección al folclor de la región.

Por los resultados de la investigación, se plantea que la principal debilidad que tiene el folclor llanero y por lo cual este no ha logrado expandirse y potencializar sus virtudes, y que por lo mismo, las futuras generaciones provenientes de los llanos orientales, vayan perdiendo el arraigo por la cultura llanera, se desprende principalmente de la falta de unión por parte de los exponentes de la región, así como lo afirma el $20 \%$ de los encuestados, los cuales no visualizan la evolución que puede llegar a tener el joropo, sino que se limitan a mantener una tradición sin salirse de los parámetros ya establecidos. Esto, sumado a la falta de articulación con el gobierno, y la carencia de estudios sobre el tema frena los intentos de promover y divulgar el folclor en mercados internacionales.

Otra de las falencias que se evidencian en los resultados es la errada divulgación sobre lo que representa el folclor llanero, la cual se les está brindando a los sucesores de las tradiciones, ya que muchas de los instructores y maestros que educan culturalmente, no lo hacen con base con una investigación pertinente de las raíces llaneras, ignorando la necesidad de investigar más la música y el baile como lo afirma Oscar Pabón, lo que da como resultado que muchos de 
los aprendices en academias ignoran cual es el contexto cultural del llanero. Se considera que esta realidad puede llegar a afectar la percepción que tiene el extranjero de lo que significa $y$ conlleva el joropo y en general la cultura de los llanos.

No obstante y quizás lo que más afecta el desarrollo y posicionamiento del folclor llanero, es la falta de apoyo por parte de los entes gubernamentales de los departamentos donde se vive la cultura llanera, adicionalmente.

Por otro lado, tras la investigación, apoyándonos en la bibliografía consultada, se considera que el aspecto más importante y fuerte de la cultura llanera es el joropo, ya que el $100 \%$ de los exponentes entrevistados declaran que el Joropo es el factor que está generando mayor impacto y reconocimiento a nivel nacional e internacional, evidenciando la buena acogida que han generado la presentaciones de algunos artistas en el exterior. Así mismo, el hecho de que la gran mayoría de las personas encuestadas, entre nacionales y extranjeros, tengan una buena percepción sobre el joropo y lo consideren motivo para atraer turistas extranjeros, hace que se dé un enfoque más claro a lo que debe trasmitir la marca.

A partir de esta información se desprende otro interrogante, al cual también se le da solución por medio de los resultados obtenidos de los instrumentos aplicados, y es el hecho de tener claridad del alcance de la palabra joropo, lo que conlleva y encierra esta representación del folclor llanero, la cual estará ligada directamente con los símbolos icónicos, cromáticos y nominales de la marca. Por esta razón, es muy importante identificar qué aspectos encierra el joropo, debido a que este es un factor clave para llevar la esencia del folclor llanero al exterior.

Según los resultados que arrojaron las encuestas a nacionales y extranjeros, así como las entrevistas a los folcloristas de renombre se cree que el joropo comprende básicamente a las representaciones artísticas musicales. En ese sentido, las entrevistas arrojaron como resultado que el $100 \%$ de los folcloristas considera que el joropo es la representación musical llanera que abarca el baile, la música y el canto. En congruencia con esto, los resultados de las encuestas evidencian la perspectiva que tienen las personas de la región sobre lo que comprende el joropo, en donde el $50 \%$ de los encuestados coinciden que el baile es un factor esencial en el joropo, seguido de los instrumentos, el canto y el trabajo de llano con un $25 \%$ cada uno.

De la misma manera, las encuestas realizadas a los extranjeros dieron como resultado que las personas que conocen que es joropo, lo perciben como una representación que incluye cantos, baile e instrumentos con un $100 \%, 92.3 \%$ y $76.9 \%$ de inclusión respectivamente. También se evidencia el $87,5 \%$ de los extranjeros encuestados ve la música y el baile como uno de los mayores atractivos para visitar la región de los llanos orientales. Esto confirma lo dicho por Miguel Ángel Martin en su libro del folclor (2000) en donde dice que el joropo "es la fiesta de los llaneros donde improvisa en cada una de sus formas de música, canto y danza".

Con base en estos resultados, se puede resumir se considera que la marca joropo debe potencializar los atributos que tiene el folclor llanero mencionados anteriormente, para que de esta manera, por medio de una buena estrategia se logre su internacionalización.

También se llega a la conclusión que el factor clave que deben tener los elementos para la creación de la marca, es el joropo, el cual, por medio de las representaciones musicales, logre representar de manera acertada la esencia del folclor llanero en el exterior.

Es necesario el señalar que los resultados de esta investigación no deben ser adjudicados a la población general ya que como se mencionó en la metodología del presente artículo, los instrumentos aplicados se hicieron como una prueba piloto para ampliación de la investigación que se está llevando a cabo en el semillero de investigación Interbranding, el cual está adscrito a la facultad de Negocios 
Internacionales de la Universidad Santo Tomás sede Villavicencio, por lo tanto, no podemos llegar a afirmaciones concluyentes en esta etapa de la investigación.

Por otro lado, de estos resultados se desprende la información que puede ser de utilidad para las entidades del gobierno que estén evaluando la posibilidad de crear una marca para el folclor llanero, así como para los folcloristas, o personas nacionales o extranjeras que deseen tener una plena identificación de sus atributos.

Esto nos plantea la necesidad de continuar con la investigación, para que de esta manera se dé solución a todos los interrogantes y objetivos planteados para que de esta manera se dé solución a todos los interrogantes y objetivos planteados para la creación de la marca.

Se sugiere que para la futura creación de una marca que represente el folclor llanero se tenga como base esta investigación debido a que en la primera fase se arrojaron resultados positivos visualizando a futuro una gran oportunidad para internacionalizar la marca y con ella la región.

\section{Referencias}

Baquero, A. (1990). "Joropo: identidad llanera, (la epopeya cultural de las comunidades del Orinoco). Llanos Orientales". Bogotá: Empresa Editorial Universidad Nacional de Colombia. Brenes, G., \& Darner, F. (2008). "Las Born Global. Empresas de Acelerada Internacionalización”. TEC Empresarial.
Marroquin, E., \& Castroman, Á. (s.f.). Internacionalización de empresas. Estudio de caso: gestión internacional de una empresa de capital latinoamericano. Obtenido de https://www.google.com.co/url?sa=t\&rct=j\&q= \&esrc $=$ s \&source $=$ web $\& \mathrm{~cd}=7 \& \mathrm{cad}=\mathrm{rja} \& u a c t=8$ \&ved=0CEUQFjAG\&url=http $\% 3 \mathrm{~A} \% 2 \mathrm{~F} \% 2 \mathrm{Fww}$ w.ead.fea.usp.br\%2FSemead\%2F6semead\%2FA DM\%2520GERAL\%2F042Adm-

\%2520Internacionaliza\%25E7ao\%2520de\%252

0Empresas.doc\&ei=qGwqVeiLLsmoNp7WgMg

$\underline{\text { M\&us }}$

Martin, M. A. (2000). "Del folclor llanero". Villavicencio: Villavicencio: Lit. Juan XXIII, sitio web de Banco de la República - Actividad Cultural, [en línea], disponible en: http://www.banrepcultural.org/blaavirtual/folclor /folclor/indice.htm

Roberts, K. (2005). "LoveMarks". Empresa Activa.

Ruiz Churrion, J. (Marz0 de 2013). "La Evolucion del Joropo". (J. A. Guzmán, Entrevistador), sitio web de YouTube, [en línea], disponible en: https://www.youtube.com/watch?v=i3eyksjBTO A 